\title{
The surgical treatment of the long head of biceps tendon and the autotenodesis phenomenon: an ultrasound and arthroscopic study
}

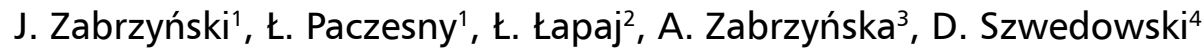 \\ ${ }^{1}$ Department of Orthopaedic Surgery, Orvit Clinic, Torun, Poland \\ 2Depertment of General Orthopaedics, Musculoskeletal Oncology and Trauma Surgery, \\ Poznan University of Medical Sciences, Poznan, Poland \\ ${ }^{3}$ Department of Radiology, Multidisciplinary Hospital, Inowroclaw, Poland \\ ${ }^{4}$ Department of Orthopaedic and Trauma, District Hospital, Torun, Poland
}

[Received: 21 May 2019; Accepted: 26 June 2019]

Background: Open or mini-invasive operative procedures are methods of choice in the treatment of the advanced degenerative process of tendinopathy of long head of biceps tendon (LHBT). Cosmetic arm deformity and fatigue are the main complaints after the surgery. Researchers have noticed that in some cases the typical cosmetic deformity is often barely noticeable and the pain is significantly reduced as it occurs after spontaneous LHBT rupture due to extremely advanced tendinopathy.

Materials and methods: This study included 41 of 75 patients who underwent LHBT arthroscopy-assisted tenotomy, followed by examination conducted by means of dedicated clinical tests, the American Shoulder and Elbow Surgeons Score (ASES) and ultrasounds.

Results: The average time interval from surgery to follow-up in the cohort was 31 months, the mean outcome measured with the ASES was 87 points and the "Popeye deformity" complication was present in 15 individuals. In the group of 26 patients where the Popeye deformity was absent and the arm contour was similar to that of the opposite arm, sonographic examination revealed the LHBT stump at the level of the intertubercular groove that was hyperechogenic and wider than the part under the groove.

Conclusions: Recent reports about the absence of the cosmetic deformity in the anterior area of the arm after shoulder arthroscopy are based on the autotenodesis phenomenon. The intra-articular part of LHBT is painlessly trapped in the bicipital groove by the surrounding soft tissues, which results in unchanged biceps muscle length; however, it is more probable to happen in patients without massive rotator cuff tears. (Folia Morphol 2020; 79, 2: 395-401)

Key words: autotenodesis, tendon, biceps, Popeye deformity, tenotomy, arthroscopy

Address for correspondence: J. Zabrzyński, MD, PhD, Orvit Clinic, ul. Marii Skłodowskiej-Curie 73, 87-100 Toruń, Poland, tel: +48 513094738, e-mail: zabrzynski@gmail.com 


\section{INTRODUCTION}

The biceps brachii muscle lies in the anterior compartment of the upper arm and has two heads: the long head arises from the superior glenoid labrum and the supraglenoid tubercle (the long head of the biceps tendon), while the short head arises from the apex of the coracoid process (the short head of the biceps tendon). These two heads join to form a single belly to be inserted to the radial tuberosity as the biceps brachii aponeurosis [19].

The function of the long head of the biceps tendon (LHBT) in the shoulder is still controversial and complex. Unfortunately, LHBT disorders are a growing problem in both athletic and non-athletic population [13]. Tendinopathy of the LHBT is one of the most common causes of chronic anterior shoulder pain, typically localised within the bicipital groove on the proximal part of humerus, 3-6 cm below the anterior margin of the acromion [28]. The role of the LHBT in anterior shoulder pain is undeniable, but - despite advances in orthopaedics - the solution of this bothering issue still poses a challenge $[2,6]$. Long head of the biceps (LHB) tendinopathy is a group of chronic tendon disorders that could be classified in multiple categories, yet three main groups are distinguished in literature and everyday practice: inflammation, instability and traumatic tendon lesions [10]. Despite complex aetiology and various clinical classifications, changes in the inner structure of the tendon are still uniform: the disruption of collagen hierarchical structure, alterations of tenocytes (local fibroblasts) and chaotic neural and vascular tissue expansion with disorders in neurotransmitters secretion [1, 9, 20].

The rotator cuff (RC) stabilises the humeral head in the normal articulated position at the glenoid fossa during the arm movements. This kind of musculo-tendinous hood is composed of the supraspinatus (SST), the infraspinatus, the teres minor and the subscapularis (SSC) tendons with concomitant, underlying fibrous capsule of the glenohumeral joint [16]. Tendinopathy of the RC is a source of pain, especially on the lateral side of the arm, and restricted active movement of the arm. The degeneration rate of the $\mathrm{RC}$ tendons increases with age. This phenomenon is starting after 50 s and is increasing, includes $50 \%$ of dominant shoulders in 70 s and $80 \%$ of shoulders in $80 \mathrm{~s}$. The SST is the most vulnerable and prone to the degeneration [27].

A large population of patients suffering from LHB tendinopathy have usually a concomitant shoulder pathology that is often primary to biceps tendon disorders, e.g. rotator cuff tears (RCTs) or superior labral tear from anterior to posterior (SLAP) [2, 13]. However, in some cases it is difficult to precisely indicate the primal lesion, especially when there is gross LHBT instability and where multiple RCTs exist. In such a situation, clinical tests are confounding and the imaging techniques are insufficient $[3,5,8$, 23]. LHB tendinopathy can simulate a RC pathology and vice versa; moreover, LHBT instability can lead to SST and SSC tears. On the other hand, SSC or SST tears expose the biceps pulley to damage and make biceps tendon prone to migration and further luxation. Similarly, as diagnostics is rather complicated, the treatment of LHB tendinopathy depends on the surgeon and no simple protocol exists in respect thereof [2].

Inflammation of the LHBT is usually transient and the treatment protocol is clear: the rest of the arm on the sling, nonsteroidal anti-inflammatory drugs and corticosteroid injections and physical therapy (kinesiotherapy: stretching, scapular muscle strengthening, restoration of full range of motion in glenohumeral joint). Surgical intervention is indicated after 3 months of initial, unsuccessful conservative treatment $[6,10]$. The acute LHBT traumatic rupture in a young and active population demands early surgical intervention with tendon tenodesis in the bicipital groove area using anchors, interference screws or tenodesis with soft tissues. On the other hand the elder population, usually $>50$ years old, does not demand surgical repair and tolerates the LHBT rupture with satisfactory clinical outcomes $[6,10]$. The LHBT instability and dislocation, usually connected with $\mathrm{RCTs}$, is predominantly an indication to operative treatment $[6,10]$.

This wide spectrum of pathologies, which can affect the LHBT, often leads to advanced tendinopathy, when the open or mini-invasive operative treatment becomes a method of choice. Although tenotomy and tenodesis are usually preferred by surgeons, the superiority of the previously mentioned procedures remains a matter of debate. Cosmetic arm deformity and fatigue are the main complaints after the tenotomy procedure [26]. However, as noticed in expert literature, typical cosmetic deformity is often barely noticeable after spontaneous LHBT rupture due to extremely advanced tendinopathy [17]. According to the reports about avoiding and minimising the occurrence of distinctive arm deformity after the ten- 
otomy procedure, we verified these conditions among arthroscopically treated patients at our department.

The aim of the study was to investigate the morphology and clinical manifestation of the autotenodesis phenomenon after shoulder arthroscopy, using ultrasounds, arthroscopy and physical examination.

\section{MATERIALS AND METHODS}

\section{General data}

The study was approved by an institutional review board and all participants provided their written informed consent prior to undertaking any study procedures (KB 62/2017). This study included 41 patients who underwent treatment in the Orthopaedic Department specialising in arthroscopic and mini-invasive surgery. The study inclusion criteria were: patient's age $\geq 25$ and $<79$ years, proximal biceps tendinopathy (tears, instability, SLAP tears) diagnosed preoperatively by means of physical examination and/or imaging techniques and confirmed during diagnostic arthroscopy. A minimum 6-month follow-up was required for inclusion. We contacted 75 patients in total and finally included 41 individuals.

Thirty-seven patients in the cohort underwent additional procedures: $\mathrm{RC}$ repair (25 patients), various types of SLAP repair (7 patients), acromio-clavicular joint resection (1 patient), anterior labrum repair (3 patients) and joint debridement due to omarthrosis (OA, 5 patients). There were only 3 patients with isolated LHB tendinopathy.

\section{Operative technique and rehabilitation}

All patients underwent shoulder arthroscopy (standard $30^{\circ}$ arthroscope $\mathrm{S} \& \mathrm{~N}$ ) in the beach-chair position with the LHBT arthroscopically-assisted tenotomy procedure and concurrent lesions repair. The standard posterior portal and additional working portals were used. For the tenotomy procedure, the proximal biceps was cut at its insertion to the labral complex (anchor); subsequently, the superior labrum was debrided (Fig. 1).

The rehabilitation protocol after the tenotomy procedure included an arm sling which was worn for 3 weeks followed by early passive and then active shoulder range of motion exercises and strength improvement $(90 \%$ of patients participated in exercises with physiotherapists from their local medical centre). Active flexion of the elbow was allowed 2 weeks post-surgery. However, if there was an additional pathology, i.e. RCTs, the rehabilitation was adjusted to this concomitant condition.

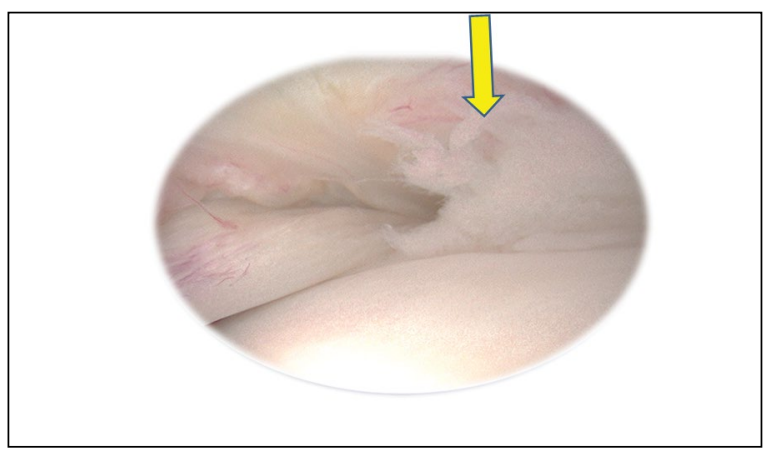

Figure 1. Arthroscopic image of long head of biceps tendon after tenotomy, entrapped in the bicipital groove (arrow).

\section{Follow-up examination}

All patients underwent physical examination by two independent orthopaedic surgeons and the clinical outcomes were assessed using: the ASES scale (the American Shoulder and Elbow Surgeons Score), clinical tests dedicated for biceps tendinopathy (Speed's test, tenderness over the bicipital groove test, Yergason test), Popeye deformity (PD) occurrence and comparison of the arm contour to that of the opposite arm [24]. All patients underwent ultrasound examination, performed by two orthopaedic surgeons experienced in musculoskeletal sonography $(13 \mathrm{MHz}$ transducer using Esaote My Lab Gamma with MSK setting). The protocol of the sonographic examination included the localisation of the LHBT stump in the bicipital groove, in the sitting position, with shoulder in $10^{\circ}$ of internal rotation, supinated elbow flexed to $90^{\circ}$ and the forearm resting on the lap. Short and long axis scans of the LHBT were taken: the probe was placed at the level of the bicipital groove, between the lesser and the greater tuberosity, and the LHBT stump in the groove, then the transducer was orientated perpendicularly between the tuberosities to obtain longitudinal scans. The stump was visualised on axial and longitudinal sonograms. To obtain superior images and eliminate the anisotropy effect, the probe was adjusted parallel to the tendon for both the transverse and longitudinal views.

\section{RESULTS}

The average time interval from surgery to follow-up in the cohort was 31 months (range 7-54). The average age at follow-up was 58 years (range 27-76). The study included 30 men and 11 women. The mean outcome measured by the ASES was 87 points (range 50-100). The PD complication was present in 15 indi- 


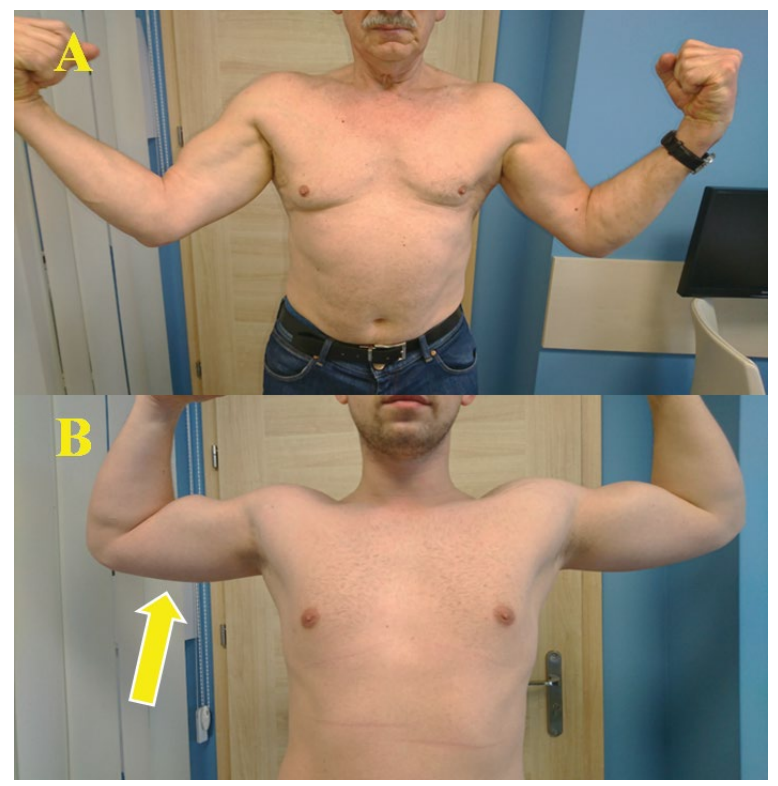

Figure 2. A. Patient after arthroscopy-assisted long head of biceps tendon (LHBT) tenotomy of the right shoulder without Popeye deformity (PD); B. Patient after arthroscopy-assisted LHBT tenotomy of the right shoulder with PD (arrow).

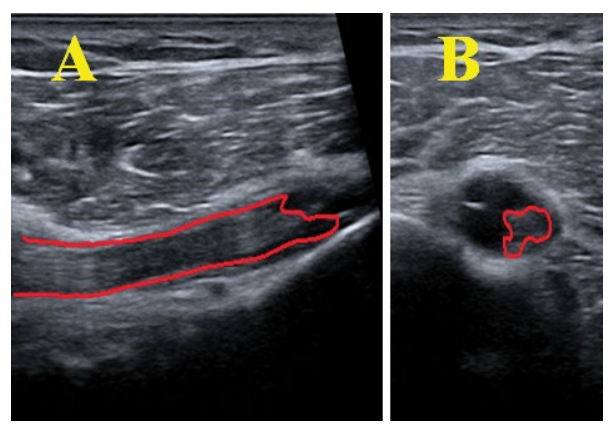

Figure 3. Ultrasound picture of long head of biceps tendon stump outside the groove; $\mathbf{A}$. The stump in the subpectoral zone was visible as a fibrillar band of the tendon (red border); B. Narrow hyperechogenic tissue localised in the intertubercular groove (red border).

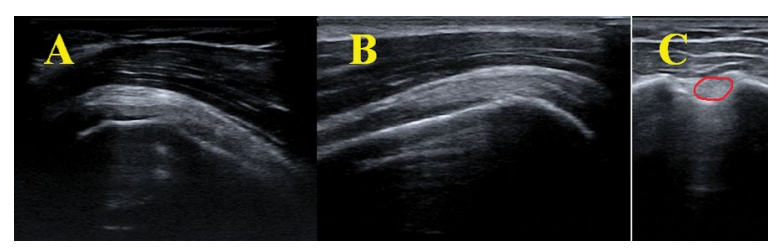

Figure 4. Ultrasound pictures. A-C. Long head of biceps tendon stump at the level of the intertubercular groove was hyperechogenic, clearly marked in the transverse and longitudinal scans (red line).

viduals; however, no patient complained on the visual appearance of arm contour. Indeed, the contour of the affected arm was enlarged when compared to the opposite side (Fig. 2A, B).
Table 1. Comparison of the autotenodesis and non-autotenodesis group of patients

\begin{tabular}{lcc}
\hline & $\begin{array}{c}\text { Autotenodesis } \\
\text { group }\end{array}$ & $\begin{array}{c}\text { Non-autotenodesis } \\
\text { group }\end{array}$ \\
\hline The average age & 57 & 59 \\
(range 27-76) & (range 45-72) \\
No. of patients & 26 & 15 \\
Sex & 11 female, & 15 male \\
& 15 male & \\
ASES score & 89 & 86 \\
Additional shoulder lesions & Various & Lesions dominated \\
lesions & byCTs \\
Positive clinical tests for & 5 & 1 \\
LHB tendinopathy & & 2 \\
Smoking & 7 & 3 \\
Painkillers & 4 & 11 \\
Return to previous activity & 20 & 0 \\
Other complications & 0 & \\
\hline
\end{tabular}

ASES - American Shoulder and Elbow Surgeons Score; LHB — long head of biceps; RCTs - rotator cuff tears

The PD was present in 12 patients who suffered from RCT - not a rare massive lesion. Other 3 patients had a SLAP I lesion, OA and isolated LHB tendinopathy, respectively. These patients had relatively modest hyperechogenic tissue localised in the intertubercular groove during the ultrasounds examination, which may correspond to the biceps pulley (Fig. 3).

Sonographically, the stump was visible below the intertubercular groove as a fibrillar band of tendon. Most patients with SLAP lesions, anterior labrum injuries, isolated LHB tendinopathy and OA had no PD. In the group of 26 patients where the PD was absent and the arm contour was similar to that of the opposite arm, the sonographic examination revealed the LHBT stump at the level of the intertubercular groove. It was hyperechogenic and wider than the lower part under the groove (Fig. 4).

The autotenodesis phenomenon was confirmed sonographically in all of the 26 individuals with absent PD. We divided the study group into the autotenodesis group and the non-autotenodesis group. Details from the examination are presented in Table 1.

\section{DISCUSSION}

A significant part of population suffers from anterior shoulder pain and LHB tendinopathy is definitely a source of this condition [6]. Pathologies affecting the LHBT are usually associated with concomitant shoulder disorders, though it is often a secondary 
process [13]. The correlation between LHBT disorders and RCTs and SLAP injuries is high and results from the fact that the biceps tendon is compensating for the muscle-tendon imbalance in shoulder joint in these pathological conditions $[6,21]$. These clinical findings associated with LHB tendinopathy have a significant impact on the treatment. Conservative treatment is usually insufficient due to rare occurrence of isolated LHBT tendinopathy and a combination of biceps pathology with different lesions of the shoulder joint, which demands operative treatment.

The LHBT originates from the superior portion of the glenoid labrum and the supraglenoid tubercle, then the intra-articular part passes antero-superiorly to the humeral head, entering the space between the greater and the lesser tuberosity of the humeral head, called the intertubercular groove, where it becomes the extra-articular part $[13,25]$. Wide and flat, the intra-articular part of the LHBT is more susceptible to degeneration due to its exposition to such forces as tear, compression and friction, whereas the extra-articular part is more rounded, smaller (tubular shape) and exposed to traction forces [9]. The LHBT has a greater diameter in its intra-articular part (particularly at the origin point) than in the extra-articular part, at the level of the bicipital groove [17]. The authors emphasised the fact that during LHBT spontaneous rupture or arthroscopic tenotomy, the stump - usually flattened and wide - is trapped in the tissue surrounding the bicipital groove consisting mainly of SSC, SST, coraco-humeral ligament and superior gleno-humeral ligament fibres [17, 22, 26]. Additionally, in the course of tendinopathy, the structure of the tendon becomes softer and the diameter increases due to the accumulation of the non-collagen extracellular matrix. Moreover, the intra-articular part of the LHBT lies extra-synovially and is covered by the sheath which acts as a "Chinese finger trap mechanism" during the tenotomy and the collapse of the tendon into the bicipital groove [17]. Several previously mentioned typical and special anatomical features of the LHBT make it possible to lock the tendon stump within the bicipital groove, resulting in the autotenodesis phenomenon. During sonographic examination in the autotenodesis group we encountered an enlarged stump at the level of the intertubercular groove as compared to the lower part of the LHBT. Contrary to these findings, the area of the intertubercular groove was filled with a modest amount of hyperechoic tissue in patients with PD, which may probably correspond to the biceps pulley.

In 1990, Patte et al. [18] observed that spontaneous rupture of the LHBT causes the analgesic effect in the affected shoulder. Next, the authors introduced the tenotomy procedure, simply releasing the tendon from its anchor, to reduce the pain. Walch et al. [26] noticed good clinical outcomes after LHBT tenotomy among elderly patients with irreparable RCTs and significant biceps tendinopathy. Moreover, Kempf et al. [11] suggested routine LHBT tenotomy in patients with RCTs. The main concerns related to this method of treatment include: an occurrence of noticeable cosmetic deformity of the arm, called "Popeye deformity", which is an abnormal shortening and bulging of the belly of the biceps muscle, pain in the anterior area of the arm and fatigue after intensive elbow flexions or exercises [14]. The term "Popeye" is related to Popeye the Sailor, a cartoon fictional character, who tensed the muscles of the forearm, not the biceps, in normal conditions - not after eating spinach. It is thought that tenotomy is generally reserved for middle-aged and older populations, who are usually non-athletic [14]. Athletic population and heavy workers are usually treated with the tenodesis procedure. We realised that tenotomy is not a surgical procedure dedicated for the entire population and demands an individual approach; on the other hand, considerable pain alleviation, early rehabilitation with wide range of motion and a low complication rate encourage the use of this method [12]. Additionally, the occurrence of the autotenodesis phenomenon after the tenotomy procedure makes it similar to the tenodesis method; however, it results from the anatomical conditions that are not completely explored. The autotenodesis phenomenon allowed the biceps muscle length to remain relatively unchanged with the reduction of pain in the anterior shoulder area [17]. On the other hand, the tenodesis procedure is often associated with some complications and the main concern is permanent pain in the implant location [13]. The important fact is that the PD was not completely prevented and autotenodesis appears randomly with no discovered pattern [12]. During the arthroscopic-assisted tenotomy procedure Osbahr et al. [17] cut the LHBT near its anchor in the glenohumeral joint, which probably led to biceps tendon being retracted and painlessly trapped in the bicipital groove. The authors additionally rec- 
ommend avoiding forceful elbow flexion for 4 weeks after arthroscopic treatment to minimise the risk of muscle deformation. We have positive experience with the mobilisation of patients and introduction of the rehabilitation protocol immediately after the procedure. Various concomitant lesions additionally complicate this protocol. An interesting fact is that there was no PD in the women's group despite the presence of massive RCTs, which could be connected with females pursuing less physical and sports activity.

As new solutions are being developed, Narvani et al. [16] introduced a novel technique for LHB tenotomy that avoids the Popeye muscle deformity. The authors incise the tendon in a special way, creating a kind of anchor in the bicipital groove. This seems to be a reasonable approach, confirming the anatomical theories of the entrapped biceps tendon stump in the intertubercular groove, surrounded the local soft tissue. Other authors described an arthroscopic technique with an idea to create a loop of the proximal portion of the LHBT, preventing the retraction below the bicipital groove [7]. The unusually rapid development of the sonography and ultrasound-guided techniques allowed Atlan et al. [4] to conduct a cadaveric study of ultrasound-guided intra-articular tenotomy with promising results.

\section{Limitations of the study}

Our study has several limitations, predominantly due to the fact that we included a moderate group of patients with a combination of concomitant shoulder lesions, but it is characteristic and typical of LHB tendinopathy. Complex injuries influence and complicate the recovery and rehabilitation protocol. Moreover, postoperative examination and outcomes may have been influenced by associated shoulder lesions. We believe that the autotenodesis phenomenon could be also disturbed in some patients by massive RCTs, particularly associated with SST and SSC, whose fibres in fact form the biceps pulley. When the pulley is inefficient, there is a minimal chance of the occurrence of the autotenodesis phenomenon. Patients with massive RCTs usually had a PD; on the other hand, the PD occurrence was less frequent and the autotenodesis phenomenon was present in patients with isolated SLAP, anterior labrum lesions, OA and isolated LHB tendinopathy. It should be noted that sonographic examination does not allow the visualisation of the entire intracapsular part of the LHBT; moreover, this technique depends highly on the device used and the observer's skills.

\section{CONCLUSIONS}

Recent reports about the absence of cosmetic deformity in the anterior area of the arm after shoulder arthroscopy are based on the autotenodesis phenomenon. Although the intra-articular part of the LHBT is painlessly trapped in the bicipital groove by the surrounding soft tissue and does not change biceps muscle length, it is more possible to happen in patients without massive rotator cuff tears.

\section{REFERENCES}

1. Abate M, Silbernagel KG, Siljeholm C, et al. Pathogenesis of tendinopathies: inflammation or degeneration? Arthritis Res Ther. 2009; 11(3): 235, doi: 10.1186/ar2723, indexed in Pubmed: 19591655.

2. Ahrens PM, Boileau P. The long head of biceps and associated tendinopathy. J Bone Joint Surg Br. 2007; 89(8): 1001-1009, doi: 10.1302/0301-620X.89B8.19278, indexed in Pubmed: 17785735.

3. Armstrong A, Teefey SA, Wu T, et al. The efficacy of ultrasound in the diagnosis of long head of the biceps tendon pathology. J Shoulder Elbow Surg. 2006; 15(1): 7-11, doi: 10.1016/j.jse.2005.04.008, indexed in Pubmed: 16414462 .

4. Atlan F, Werthel JD. Ultrasound-guided intra-articular tenotomy of the long head of the biceps: a cadaveric feasibility study. Int Orthop. 2016; 40(12): 2567-2573, doi: 10.1007/s00264-016-3231-2, indexed in Pubmed: 27262991.

5. Carr RM, Shishani Y, Gobezie R. How accurate are we in detecting biceps tendinopathy? Clin Sports Med. 2016; 35(1): 47-55, doi: 10.1016/j.csm.2015.08.002, indexed in Pubmed: 26614468.

6. Ditsios K, Agathangelidis F, Boutsiadis A, et al. Long head of the biceps pathology combined with rotator cuff tears. Adv Orthop. 2012; 2012: 405472, doi: 10.1155/2012/405472, indexed in Pubmed: 23209915.

7. Goubier JN, Bihel T, Dubois E, et al. Loop biceps tenotomy: an arthroscopic technique for long head of biceps tenotomy. Arthrosc Tech. 2014; 3(4): e427-e430, doi: 10.1016/j. eats.2014.04.005, indexed in Pubmed: 25264503.

8. Harpreet S, El Ra, Bahk MS, et al. Physical examination for partial tears of biceps tendon. Am J Sports Med. 2007; 35(8): 1334-40.

9. Joseph M, Maresh CM, McCarthy MB, et al. Histological and molecular analysis of the biceps tendon long head post-tenotomy. J Orthop Res. 2009; 27(10): 1379-1385, doi: 10.1002/jor.20868, indexed in Pubmed: 19340876.

10. Khazzam M, George MS, Churchill RS, et al. Disorders of the long head of biceps tendon. J Shoulder Elbow Surg. 2012; 21(1): 136-145, doi: 10.1016/j.jse.2011.07.016, indexed in Pubmed: 22005126.

11. Kempf JF, Gleyze P, Bonnomet F, et al. A multicenter study of 210 rotator cuff tears treated by arthroscopic acromioplasty. Arthroscopy. 1999; 15(1): 56-66, doi: 10.1053/ ar.1999.v15.015005.

12. Kelly AM, Drakos MC, Fealy S, et al. Arthroscopic release of the long head of the biceps tendon: functional outcome and clinical results. Am J Sports Med. 2005; 33(2): 
208-213, doi: 10.1177/0363546504269555, indexed in Pubmed: 15701606.

13. Longo UG, Loppini M, Marineo G, et al. Tendinopathy of the tendon of the long head of the biceps. Sports Med Arthrosc Rev. 2011; 19(4): 321-332, doi: 10.1097/ JSA.0b013e3182393e23, indexed in Pubmed: 22089281.

14. Meeks BD, Meeks NM, Froehle AW, et al. Patient satisfaction after biceps tenotomy. Orthop J Sports Med. 2017; 5(5): 2325967117707737, doi: 10.1177/2325967117707737, indexed in Pubmed: 28596975.

15. Naidoo N, Lazarus L, Osman SA, et al. An arthroscopic evaluation of the anatomical "critical zone". Folia Morphol. 2017; 76(2): 277-283, doi: 10.5603/FM.a2016.0049, indexed in Pubmed: 27665956.

16. Narvani AA, Atoun E, Van Tongel A, et al. The "anchor shape" technique for long head of the biceps tenotomy to avoid the popeye deformity. Arthrosc Tech. 2013; 2(2): e167-e170, doi: 10.1016/j.eats.2013.01.008, indexed in Pubmed: 23875145.

17. Osbahr DC, Diamond AB, Speer KP. The cosmetic appearance of the biceps muscle after long-head tenotomy versus tenodesis. Arthroscopy. 2002; 18(5): 483-487, doi: 10.1053/jars.2002.32233, indexed in Pubmed: 11987057.

18. Patte $D$, Walch G, Boileau P. Luxation de la longue portion du biceps et rapture de la cauffe des rotateurs. Revue de Chirurgie Orthopedique. 1990; 76: 95.

19. Piagkou M, Totlis T, Anastasopoulos N, et al. An atypical biceps brachii and coracobrachialis muscles associated with multiple neurovascular aberrations: a case report with clinical significance. Folia Morphol. 2019; 78(2): 444-449, doi: 10.5603/FM.a2018.0081, indexed in Pubmed: 30178460 .

20. Pingel J, Lu $Y$, Starborg T, et al. 3-D ultrastructure and collagen composition of healthy and overloaded human tendon: evidence of tenocyte and matrix buckling. J Anat. 2014; 224(5): 548-555, doi: 10.1111/joa.12164, indexed in Pubmed: 24571576.

21. Sakurai G, Ozaki J, Tomita Y, et al. Morphologic changes in long head of biceps brachii in rotator cuff dysfunc- tion. J Orthop Sci. 1998; 3(3): 137-142, doi: 10.1007/ s007760050033, indexed in Pubmed: 9683766.

22. Singh $R$, Singla $M$, Tubbs RS. Macro/micro observational studies of fibres maintaining the biceps brachii tendon in the bicipital groove: application to surgery, pathology and kinesiology. Folia Morphol. 2015; 74(4): 439-446, doi: 10.5603/FM.2015.0105, indexed in Pubmed: 26620503.

23. Skendzel JG, Jacobson JA, Carpenter JE, et al. Long head of biceps brachii tendon evaluation: accuracy of preoperative ultrasound. Am J Roentgenol. 2011; 197(4): 942-948, doi: 10.2214/AJR.10.5012, indexed in Pubmed: 21940583.

24. Ślęzak M, Lubiatowski P, Lubiatowski B, et al. Polish cultural adaptation and validation of patient self-assessment questionnaires for painful shoulder: ASES, SST, Constant Score and UCLA. Issue Rehabil Orthop Neurophysiol Sport Promot. 2016; 17.

25. Vangsness $\mathrm{CT}$, Jorgenson SS, Watson $\mathrm{T}$, et al. The origin of the long head of the biceps from the scapula and glenoid labrum. An anatomical study of 100 shoulders. J Bone Joint Surg Br. 1994; 76(6): 951-954, indexed in Pubmed: 7983126.

26. Walch G, Edwards TB, Boulahia A, et al. Arthroscopic tenotomy of the long head of the biceps in the treatment of rotator cuff tears: clinical and radiographic results of 307 cases. J Shoulder Elbow Surg. 2005; 14(3): 238-246, doi: 10.1016/j.jse.2004.07.008, indexed in Pubmed: 15889020.

27. Zabrzyński J, Łapaj $\npreceq$, Paczesny $k$, et al. Tendon function-related structure, simple healing process and mysterious ageing. Folia Morphol. 2018; 77(3): 416-427, doi: 10.5603/FM.a2018.0006, indexed in Pubmed: 29345715.

28. Zabrzyński J, Paczesny $Ł$, Łapaj $Ł$, et al. Process of neovascularisation compared with pain intensity in tendinopathy of the long head of the biceps brachii tendon associated with concomitant shoulder disorders, after arthroscopic treatment. Microscopic evaluation supported by immunohistochemical. Folia Morphol. 2018; 77(2): 378-385, doi: 10.5603/ FM.a2017.0093, indexed in Pubmed: 29064550. 